04

\title{
Объем когерентности оптического волнового поля с широкими частотным и угловым спектрами
}

\author{
(ㄱ Д.В. Лякин, Н.Ю. Мысина, В.П. Рябухо \\ Институт проблем точной механики и управления РАН, \\ 410028 Саратов, Россия \\ ฯ e-mail: rvp-optics@yandex.ru \\ Поступила в редакцию 12.09.2017 г. \\ В окончательной редакции 02.11.2017 г.
}

\begin{abstract}
Рассмотрены размеры области трехмерного пространства, в которой оптическим волновым полем возбуждаются взаимно когерентные возмущения. Обсуждены условия, когда длина этой области вдоль направления распространения волнового поля и соответственно величина ее объема определяются или шириной частотного спектра поля, или шириной его углового спектра, или одновременно параметрами этих спектров. Получены выражения для оценки предельно малых величин объема когерентности полей с широким частотным спектром и предельно широким угловым спектром. С использованием представления о мгновенной спекл-модуляции волнового поля дается физическая интерпретация возникновения ограниченного объема когерентности поля. Определена длина области пространственно-временно́й когерентности, в которой разновременно возникают взаимно когерентные возмущения. Рассмотрен объем когерентности освещающего объект волнового поля в высокоразрешающей микроскопии в частотно широкополосном свете. Дана оценка условий доминирующего влияния углового или частотного спектров на продольную длину области когерентности, а также рассмотрены условия влияния ширины частотного спектра на поперечную когерентность волнового поля. Показано, что такое влияние требует учета при использовании в высокоразрешающей микроскопии полей с широкими и ультраширокими частными спектрами.
\end{abstract}

DOI: $10.21883 /$ OS.2018.03.45657.199-17

\section{Введение}

В теории когерентности оптических волновых полей [1-5] рассматривается объем когерентности как область трехмерного пространства, в любой паре точек которой возбуждаются в той или иной степени взаимно когерентные колебания возмущений поля. Представление об области пространственной когерентности и величине ее объема имеет не только важное феноменологическое и методологическое значение, но также и прикладное значение в физической оптике: в интерферометрии с использованием частично когерентного света [6,7], в анализе оптических систем частично когерентного формирования изображений $[8,9]$, в интерференционной микроскопии $[10,11]$ и голографической микроскопии $[12,13]$ в частично когерентном свете, в оптической когерентной томографии $[14,15]$, в системах когерентного рассеяния света в случайно неоднородных средах и формирования спекл-структур в частично когерентном свете [16,17], в теории взаимодействия оптического излучения и биологических сред $[18,19]$ и во многих других когерентно оптических методах и системах прикладной оптики.

Объем когерентности определяется как некоторая область пространства, длина которой $L_{c}$ вдоль направления распространения волнового поля ограничивается длиной продольной когерентности, а поперечный размер длинами поперечной пространственной когерентности поля $\rho_{\perp x}$ и $\rho_{\perp y}$ в ортогональных направлениях. При этом длина продольной когерентности полагается рав- ной длине временно́й когерентности $l_{c}, L_{c} \approx l_{c}[1-3]$. Такое представление о длине области когерентности вполне обоснованно для направленных пучков света волновых полей с достаточно узким угловым спектром в той области пространства, где рассматривается область когерентности поля. Однако для волновых полей с широким угловым спектром в это представление об объеме когерентности поля необходимо внести коррективы, связанные с зависимостью длины продольной когерентности волнового поля от ширины углового спектра, а также связанные с зависимостью длины поперечной когерентности от ширины частотного спектра.

В работах [20-32] теоретически и экспериментально установлено, что длина продольной когерентности волнового поля может ограничиваться не только шириной его частотного спектра, но и шириной его углового спектра или совместно и шириной частотного, и шириной углового спектров поля [33,34]. Более того, длина поперечной когерентности также оказывается зависимой от ширины частотного спектра при достаточно большой его ширине [34,35]. Вышеприведенное определение трехмерной области когерентности волнового поля следует отнести к представлению об области одновременной когерентности волновых возмущений, возбуждаемых полем в различных точках пространства в одни и те же моменты времени. Кроме одновременной когерентности имеется необходимость рассматривать область разновременной когерентности, в которой разновременно, т.е. в различные моменты времени, возбуждаются в 
определенной степени взаимно когерентные возмущения в процессе их распространения. В этом случае следует говорить об области и объеме пространственно-временно́й когерентности волнового поля.

В настоящей работе рассматривается конкурирующее влияние параметров углового и частотного спектров волнового поля на размеры и соответственно на объем трехмерной области пространственной и пространственно-временно́й когерентности волнового поля, в которых возникают одновременно или разновременно в определенной степени взаимно когерентные возмущения.

\section{Трехмерная область пространственной когерентности}

Представления о трехмерной области когерентности оптического волнового поля и ее объеме с необходимостью связаны с представлениями о пространственной поперечной и продольной когерентности поля. Под пространственной когерентностью понимается взаимная когерентность возмущений, одновременно возбуждаемых полем в двух произвольно расположенных точках трехмерного пространства. Трехмерную область пространства, в любой паре точек которой волновым полем одновременно возбуждаются колебания, имеющие степень взаимной когерентности не ниже некоторой установленной величины, следует рассматривать как объем когерентности волнового поля.

Строго одночастотное волновое поле в силу детерминированности создаваемых им возмущений гармонического характера полностью когерентно и во времени, и в пространстве. Это представление относится и к рассеянным волновым полям, если рассматривается одна реализация рассеянного поля, а не его ансамбль. Временна́я и пространственная декогерентность возмущений поля и соответственно ограниченная в пространстве область когерентности в первую очередь возникают вследствие неодночастотности возмущений, определяемых конечной шириной частотного спектра возмущений поля.

Для пространственно когерентного источника света, когда длина когерентности поля на поверхности источника превышает размеры излучающей поверхности, например для квазиточечного источника света, поперечная длина области когерентности дифракционного волнового поля $\rho_{\perp}$ ограничивается только поперечной апертурой поля - апертурой пучка света, а длина продольной когерентности $L_{c}$, определяющая длину области когерентности поля в направлении его распространения, ограничивается длиной временно́й когерентности $l_{c}, L_{c} \approx l_{c}$, зависящей от ширины $\Delta \omega$ частотного спектра возмушений поля, $l_{c} \approx c 2 \pi / \Delta \omega \approx \lambda_{0}^{2} / \Delta \lambda$, где $c-$ скорость света, $\lambda_{0}-$ средняя длина волны, $\Delta \lambda-$ ширина частотного спектра в шкале длин волн [1-5].

Пространственная частичная некогерентность протяженного источника света (а точнее, в общем случае конечная ширина углового спектра волнового поля $2 \theta$ в точке наблюдения) приводит к ограничению длины поперечной когерентности $\rho_{\perp}$ дифракционного поля и соответственно к ограничению поперечных размеров области когерентности, которые могут стать существенно меньше апертуры пучка света при достаточно широком угловом спектре волнового поля. Волновое поле становится частично когерентным и в продольном, и в поперечном направлениях.

При относительно малой угловой апертуре волнового поля $2 \theta$ поперечная длина когерентности $\rho_{\perp}$ определяется соотношением [1-3]

$$
\rho_{\perp}=\frac{\lambda_{0}}{2 \sin (\theta)} \approx \frac{\lambda_{0}}{2 \theta},
$$

а продольная длина когерентности - длиной временно́й когерентности $l_{c}, L_{c} \approx l_{c}$. Тогда объем области когерентности определяется известным выражением [1-3]

$$
V_{c} \approx \frac{\pi}{4} \rho_{\perp}^{2} l_{c} \approx \frac{\pi}{16} \frac{\lambda_{0}^{4}}{\theta^{2} \Delta \lambda}
$$

Это выражение, как правило, и используется для оценки объема когерентности оптических волновых полей во многих оптических системах, в которых формируются направленные пучки света с малой угловой апертурой и достаточно широким частотным спектром.

Однако длина $L_{c}$ продольной когерентности оптического волнового поля, как показано в [25-30,33,34], при определенных условиях, определяемых соотношением параметров частотного и углового спектров, может ограничиваться не шириной частотного спектра поля $\Delta \omega$, а шириной его углового спектра $2 \theta$ :

$$
L_{c} \approx \rho_{\|}=\frac{\lambda_{0}}{2 \sin ^{2}\left(\frac{\theta}{2}\right)} \approx \frac{2 \lambda_{0}}{\theta^{2}},
$$

где так же, как и в (1), использовано приближение малости угла $\theta, \sin (\theta / 2) \approx \theta / 2$ (параксиальное приближение). Ниже устанавливаются соотношения между параметрами частотного и углового спектров, при которых длина области когерентности в продольном направлении определяется преимущественно либо шириной частотного спектра, либо шириной углового спектра поля, не прибегая к параксиальному приближению.

При сравнимых величинах длины временно́й когерентности $l_{c}$ и длины продольной когерентности $\rho_{\|}$, определяемой в соответствии с (3) шириной углового спектра поля, ширина частотного $\Delta \omega$ и ширина углового $2 \theta$ спектров поля оказывают конкурирующее влияние на его результирующую продольную когерентность и соответственно на результирующую длину продольной когерентности $L_{c}$. В $[33,34]$ в приближении равномерного углового и равномерного частотного спектров волнового поля получено соотношение, отражающее эту конкуренцию частотного и углового спектров поля:

$$
\frac{1}{L_{c}} \approx \frac{\Delta \lambda}{\lambda_{0}^{2}} \cos ^{2}\left(\frac{\theta}{2}\right)+\frac{2}{\lambda_{0}} \sin ^{2}\left(\frac{\theta}{2}\right) \approx \frac{1}{l_{c}}+\frac{1}{\rho_{\|}},
$$




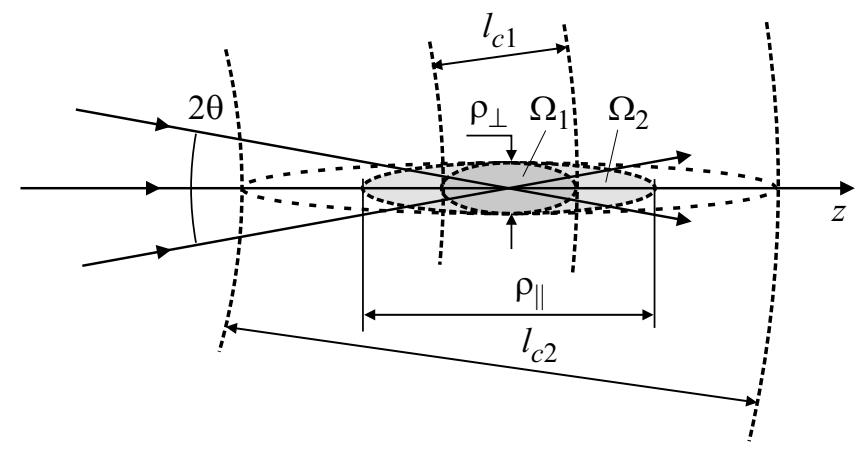

Рис. 1. Области когерентности $\Omega_{1}$ и $\Omega_{1}$ волнового поля с конечной шириной углового спектра $\theta$ и шириной частотного спектра $\Delta \omega$ при различных соотношениях длины временно́й когерентности $l_{c}$ и длины когерентности $\rho_{\|}$, определяемой шириной углового спектра поля: $\Omega_{1}$ при $l_{c}=l_{c 1} \ll \rho_{\|}, \Omega_{2}$ при $l_{c}=l_{c 2} \gg \rho_{\|}$.

откуда следует выражение для длины продольной когерентности

$$
L_{c} \approx \frac{\rho_{\|} l_{c}}{\rho_{\|} \cos ^{2}(\theta / 2)+l_{c}} \approx \frac{\rho_{\|} l_{c}}{\rho_{\|}+l_{c}} .
$$

Таким образом, при сравнимых величинах $l_{c}$ и $\rho_{\|}$ результирующая длина продольной когерентности $L_{c}$ оказывается „чувствительной“ одновременно к ширине частотного $\Delta \omega$ и ширине углового $2 \theta$ спектров волнового поля. В выражениях (4) и (5) можно пренебречь сомножителем $\cos ^{2}(\theta / 2)$ при $\Delta \lambda \ll \lambda_{0}[34,35]$. Более подробно данный вопрос рассмотрен ниже в специальном разделе настоящей работы.

В [34,35] также показано, что длина поперечной когерентности $R_{\perp}$ частотного широкополосного волнового поля становится зависимой от ширины его частотного спектра $\Delta \lambda(\Delta \omega)$ и соответственно от длины временно́й когерентности $l_{c}$, когда $\Delta \lambda \approx \lambda_{0}$,

$$
\frac{1}{R_{\perp}} \approx\left(\frac{\Delta \lambda}{\lambda_{0}^{2}}+\frac{2}{\lambda_{0}}\right) \sin (\theta)=\frac{1}{l_{c}} \sin (\theta)+\frac{1}{\rho_{\perp}},
$$

где $\rho_{\perp}$ определяется соотношением (1). Из выражения (6) следует выражение для длины поперечной когерентности

$$
R_{\perp} \approx \frac{\lambda_{0}^{2}}{\left(2 \lambda_{0}+\Delta \lambda\right) \sin (\theta)}=\frac{\rho_{\perp} l_{c}}{\rho_{\perp} \sin (\theta)+l_{c}}
$$

При $\Delta \lambda \ll \lambda_{0}$ длина поперечной когерентности определяется практически только шириной углового спектра поля, $R_{\perp} \approx \rho_{\perp}$.

Выражение (5) показывает, что если ширина частотного спектра $\Delta \omega$ достаточно большая, а угловой спектр поля достаточно узкий (угол $\theta$ мал), так что $l_{c}=l_{c 1} \ll \rho_{\|}$, то длина продольной когерентности поля $L_{c}$ определяется шириной частотного спектра $\Delta \omega$ и приблизительно равна длине временной когерентности $l_{c}$, и поэтому длина объемной области когерентности ограничивается длиной $l_{c}$ (рис. 1 , область $\Omega_{1}$ ). В этом случае формируется объем когерентности, величина которого оценивается выражением (2). Если же волновое поле в области наблюдения имеет достаточно широкую угловую апертуру $2 \theta$ при достаточно малой ширине частотного спектра $\Delta \omega$, так что $l_{c}=l_{c 2} \gg \rho_{\|}$, то длина продольной когерентности $L_{c}$ определяется шириной углового спектра $\theta$ и приблизительно равна $\rho_{\|}$, $L_{c} \approx \rho_{\|}$, и поэтому длина объемной области когерентности ограничивается длиной $\rho_{\|}$(рис. 1 , область $\Omega_{2}$ ). В этом случае формируется волновое поле, объем области когерентности которого будет определяться соотношением

$$
V_{c} \approx \frac{\pi}{4} \rho_{\perp}^{2} \rho_{\|} \approx \frac{\pi}{4}\left(\frac{\lambda_{0}}{2 \sin (\theta)}\right)^{2}\left(\frac{\lambda_{0}}{2 \sin ^{2}(\theta / 2)}\right) \approx \frac{\pi}{8} \frac{\lambda_{0}^{3}}{\theta^{4}},
$$

где использовано приближение малости угла $\theta$, $\sin (\theta) \approx \theta$ (параксиальное приближение).

Предельно малый объем когерентности $V_{\min } \approx \pi \lambda_{0}^{3} / 16$, как это следует из (8), возникает при максимальной ширине углового спектра, когда $\theta \approx 90^{\circ}$ и в волновом поле формируются стоячие волны. Такое поле, например, формируется вблизи протяженного пространственно некогерентного ( $\delta$-коррелированного) источника света или внутри объемной сильно рассеивающей среды.

В общем случае при произвольных соотношениях длин временно́й когерентности $l_{c}$ и продольной когерентности $\rho_{\|}$(при произвольных ширинах частотного и углового спектров) объем когерентности в соответствии с (5) и (7) определяется с помощью выражения

$$
\begin{aligned}
V_{c} \approx \frac{\pi}{4} & R_{\perp}^{2} L_{c} \approx \frac{\pi}{4}\left(\frac{\rho \perp l_{c}}{\rho_{\perp} \sin (\theta)+l_{c}}\right)^{2} \\
& \times \frac{\rho_{\|} l_{c}}{\rho_{\|} \cos ^{2}(\theta / 2)+l_{c}} \approx \frac{\pi}{8} \frac{\lambda_{0}^{3}}{\theta^{4}\left(1+\frac{2}{\theta^{2}} \frac{\Delta \lambda}{\lambda_{0}}\right)},
\end{aligned}
$$

где использованы приближения $\Delta \lambda \ll \lambda_{0}$ и $\sin (\theta) \approx \theta$.

При достаточно малой ширине частотного спектра $\Delta \lambda$ и сравнительно большой величине угловой апертуры поля $2 \theta$, когда $\Delta \lambda / \lambda_{0} \ll \theta^{2} / 2$, что эквивалентно $\rho_{\|} \ll l_{c}$, выражение (9) для объема когерентности переходит в (8). И наоборот, при $2 \Delta \lambda / \theta^{2} \gg \lambda_{0}$, что имеет место, когда $\rho_{\|} \gg l_{c},(9)$ преобразуется в $(2)$.

При приблизительном равенстве длин когерентности $l_{c}$ и $\rho_{\|}$, когда $\Delta \lambda \approx \lambda_{0} 2 \sin ^{2}(\theta / 2) \approx \lambda_{0} \theta^{2} / 2$, длина области когерентности $L_{c}$, как это следует из (5), составляет примерно половину $l_{c}$ или $\rho_{\|}$, и объем когерентности сокращается примерно в 2 раза,

$$
V_{c} \approx \frac{\pi}{64} \frac{\lambda_{0}^{2}}{\sin ^{2}(\theta) \sin ^{2}(\theta / 2)} \approx \frac{\pi}{16} \frac{\lambda_{0}^{3}}{\theta^{4}} \approx \frac{\pi}{32} \frac{\lambda_{0}^{4}}{\theta^{2} \Delta \lambda} .
$$


В этих условиях предельно малый объем когерентности, формируемый при максимально большом апертурном угле $\theta \approx 90^{\circ}$, становится приблизительно равным $V_{\min } \approx \pi \lambda_{0}^{3} / 32$.

\section{Физическая интерпретация возникновения ограниченного объема когерентности волнового поля}

Физические механизмы ограничения длины области когерентности волнового поля при его широком угловом спектре и узком частотном спектре, когда $l_{c} \gg \rho_{\|}$, и при его узком угловом спектре и широком частотном спектре, когда $l_{c} \ll \rho_{\|}$, имеют отличия.

В волновом поле при $l_{c} \gg \rho_{\|}$длина области когерентности ограничивается не длиной $l_{c}$ волнового цуга возмущений поля, а пространственными флуктуациями этого поля, обусловленными интерференцией его волновых компонент с различными угловыми направлениями распространения. В течение времени когерентности возмущений поля $\tau_{c} \approx 2 \pi / \Delta \omega$ разность фаз этих волновых компонент не изменяется больше, чем на $2 \pi \mathrm{rad}$, т.е. остается приблизительно постоянной, и эти волны складываются в разных точках пространства с теми или иными фазовыми соотношениями, увеличивая или уменьшая амплитуду суммарного возмущения поля. Вследствие этого возникает пространственная стохастическая амплитудно-фазовая модуляция волнового поля, которая аналогична динамической спекл-модуляции когерентного (в частности, лазерного) излучения, рассеянного на изменяющихся во времени неоднородностях среды [17,36-38]. Эта модуляция поля стохастически изменяется во времени смена реализаций спекл-модуляции происходит за время когерентности $\tau_{c}$. На рис. 2, $a, b$ представлены пространственные распределения мгновенной интенсивности (квадрата модуля возмущений поля без его усреднения по времени) в поперечном $(x, y)$ и продольном $(x, z)$ сечениях одной из возможных реализаций такого спекл-модулированного поля, полученной в результате численного моделирования. Градациями серого на этих изображениях отображены пространственные изменения мгновенной интенсивности возмущений поля. Области с повышенной мгновенной интенсивностью и соответственно большой амплитудой возмущений (поперечные и продольные спеклы поля) на изображениях представлены светлыми. Объемные спеклы каждой реализации поля в определенном смысле визуализируют область когерентности волнового поля - в спеклах поля случайные составляющие амплитуды и фазы поля претерпевают сравнительно малые пространственные изменения. Возмущения волнового поля распространяются в продольных спеклах с фазовой скоростью и пробегают их за время $\Delta t$, меньшее, чем время когерентности $\tau_{c}$, поскольку предполагается, что $l_{c} \gg \rho_{\|}$. При этом возмушения, пробежавшие длину спекла $\rho_{\|}$, приобрета-
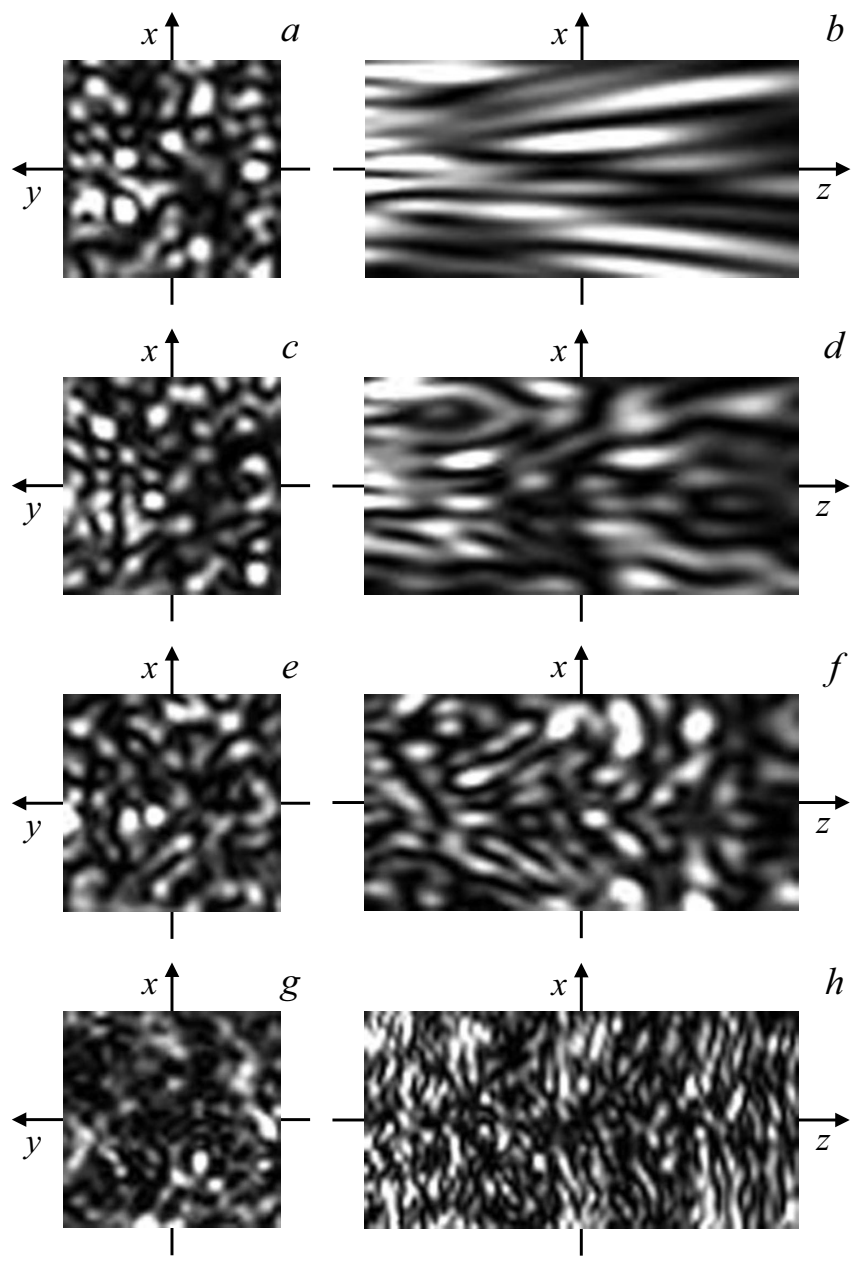

Рис. 2. Пространственные распределения мгновенной интенсивности возмущений в поперечном $x, y(a, c, e, g)$ и продольном $x, z(b, d, f, h)$ сечениях спекл-модулированной реализации волнового поля $\left(\lambda_{0}=0.63 \mu \mathrm{m}\right)$ с разной длиной временно́й когерентности $l_{c}$ по отношению к длине продольной когерентности $\rho_{\|}$, определяемой угловым спектром поля: $a, b-l_{c} \approx 27 \rho_{\|}$ $\left(\Delta \lambda=0.002 \lambda_{0}\right) ; \quad c, d-l_{c} \approx 0.27 \rho_{\|} \quad\left(\Delta \lambda=0.2 \lambda_{0}\right) ; \quad e, f-$ $l_{c} \approx 0.13 \rho_{\|}\left(\Delta \lambda=0.4 \lambda_{0}\right) ; g, h-l_{c} \approx 0.06 \rho_{\|}\left(\Delta \lambda=0.93 \lambda_{0}\right)$.

ют фазовый набег, случайная составляющая которого примерно равна $2 \pi \mathrm{rad}$, так что за промежуток времени, существенно меньший времени когерентности $\tau_{c}$, возникает полная декогерентность возмущений поля в точках, расположенных на расстоянии $\Delta z$, равном или превышающем $\rho_{\|}$, но, важно подчеркнуть, на расстоянии $\Delta z$, меньшим, чем длина временной когерентности $l_{c}, \rho_{\|} \leq \Delta z<l_{c}$.

Волновое поле с достаточно широким частотным спектром и относительно узким угловым спектром, когда $l_{c} \ll \rho_{\|}$, имеет область когерентности, длина которой ограничивается длиной временной когерентности $l_{c}$ - длиной волнового цуга колебаний поля [1-3]. Следующие друг за другом в пространстве и во времени волновые цуги отличаются не только случайной фазой, но и случайной амплитудой, плавно и, как 
правило, монотонно изменяющейся в пределах одного цуга (см., например, иллюстрации рис. 1.1 в [3] и рис. 2 в $[39,40])$. В поперечном сечении поля в силу конечности ширины углового спектра поля (конечных размеров источника) возникают пространственные амплитудно-фазовые флуктуации поля, которые определяют ограниченную поперечную когерентность поля с длиной поперечной когерентности $R_{\perp}$. Поэтому пространственное распределение мгновенной интенсивности в поперечном сечении такого волнового поля с $l_{c} \ll \rho_{\|}$будет иметь спекл-модулированный характер, подобный представленному на рис. 2,a. В продольном направлении поля (в направлении его распространения) также возникают пространственные амплитуднофазовые флуктуации, подобные спекл-модуляции, но они связаны с конечной длиной волновых цугов поля, а не с конечной шириной углового спектра поля. Таким образом, пространственные распределения мгновенной интенсивности волнового поля в его поперечном $(x, y)$ и продольном $(x, z)$ сечениях в этом случае имеют вид, аналогичный представленным на рис. 2, $a, b$. Изображения поперечных и продольных сечений этих мгновенных спекл-модулированных волновых полей представлены на рис. 2, $c-h$.

Длина образующихся объемных спеклов в продольном сечении поля в случае $l_{c} \ll \rho_{\|}$ограничивается длиной волнового цуга - длиной временной когерентности $l_{c}$, определяемой шириной частотного спектра поля, а в поперечном направлении - длиной поперечной когерентности $R_{\perp}$, определяемой преимущественно шириной углового спектра поля $2 \theta, R_{\perp} \approx \rho_{\perp}$. Благодаря этому отличается и характер динамики пространственновременных изменений этих спекл-модулированных распределений. В первом случае, при $l_{c} \gg \rho_{\|}$, смена пространственных реализаций поля и соответственно поперечных и продольных спеклов поля происходит в виде стохастического бурления (кипения) - объемные спеклы исчезают в одном месте и возникают в другом по истечении времени когерентности $\tau_{c}$. Во втором случае, при $l_{c} \ll \rho_{\|}$, объемные спеклы поля смещаются как целое в пространстве с групповой скоростью $v_{g}$, оставаясь почти неизменными („замороженными“ [41]) на пути, равном длине когерентности $\rho_{\|}$. Пройдя этот путь, спеклы испытывают стохастические амплитуднофазовые изменения - одни спеклы исчезают и возникают другие. Время этой декорреляции объемных волновых цугов $\tau_{c g} \approx \rho_{\|} / \nu_{g}$. Однако в поперечном сечении поля смещение объемных спеклов вызывает такое же бурление и смену реализаций за время когерентности $\tau_{c}$, как и в первом случае, поскольку одни спеклы уходят из этого сечения, а другие приходят. В продольном сечении продольные спеклы бегут с групповой скоростью, а отдельные возмущения поля в них - с фазовой скоростью. Длина когерентного пробега спеклов и отдельных возмущений поля ограничивается длиной продольной когерентности $\rho_{\|}$, определяемой шириной углового спектра поля $2 \theta[42,43]$.

\section{Область пространственно-временно́й когерентности}

Продольное смещение „замороженных“ мгновенных спеклов в волновом поле с $l_{c} \ll \rho_{\|}$в пределах длины когерентности $\rho_{\|}$с точки зрения корреляционных представлений определяет продольное смещение всего объема когерентности поля. В результате таких смещений мгновенных спеклов возникают корреляции возмущений поля в разные моменты времени на расстояниях $\Delta z$, превышающих длину временной когерентности $l_{c}$, но не превышающих длину когерентности $\rho_{\|}, l_{c}<\Delta z<\rho_{\|}$. Волновые возмущения, формируемые в результате сложения всех угловых компонент волнового поля, не испытывают существенных декорреляционных изменений при распространении на расстояние $\Delta z<\rho_{\|}$. На расстояниях $\Delta z \geq \rho_{\|}$возникают существенные декорреляционные изменения возмущений. Следовательно, в случае, когда $l_{c} \ll \rho_{\|}$, длина продольной когерентности $\rho_{\|}$является длиной пространственно-временной когерентности и определяет длину области пространственно-временно́й когерентности волнового поля. В этой области взаимная когерентность возмущений поля возникает не одновременно, а разновременно на расстоянии $\Delta z<\rho_{\|}$ с временным интервалом, определяемым временем распространения возмущений в продольном направлении $\tau_{c g} \approx \Delta z / \nu_{g}$.

\section{Объем когерентности в микроскопии}

Во многих практических оптических системах формируются в значительной степени направленные волновые поля, имеющие относительно узкий угловой спектр и соответственно большую длину продольной когерентности $\rho_{\|}$, определяемую, согласно (3), шириной этого спектра. Поэтому эффект продольной декогерентности, связанный с угловым спектром поля, как правило, не наблюдается в таких оптических системах. Волновые поля с достаточно широким угловым спектром часто формируются в изображающих оптических системах, в особенности в высокоразрешающей оптической микроскопии, включая интерференционную микроскопию $[10,11,15,30,44-46]$ и полнопольную когерентную томографию $[15,42,47,48]$, в которых применяются объективы и осветительные системы с большой угловой апертурой. При использовании в микроскопии протяженных и частотно широкополосных источников света освещающее объект волновое поле может иметь предельно малый для экспериментальной практики объем пространственной когерентности, определяемый угловой апертурой объектива или осветительной системы, а также шириной частотного спектра волнового поля. В таких интерференционных системах наблюдаются эффекты продольной декогерентности, связанные с широким угловым спектром волнового поля $[10,11,15,30,42,43,45-53]$. 
В интерференционной микроскопии в частично когерентном свете и полнопольной когерентной томографии ограниченный объем когерентности предопределяет условия формирования интерференционного изображения и пространственную локализацию интерференционного поля. Для обеспечения взаимной когерентности опорного и объектных волновых полей необходимо, чтобы их наложение на выходе интерференционного микроскопа происходило с взаимным пространственным смещением, не превышающим длин пространственной когерентности полей, определяющих объем когерентности [54].

В микроскопии на отражение микрообъектив микроскопа выполняет роль части осветительной системы, и угловая апертура $\theta$ освещающего объект волнового поля определяется размером $D^{\prime}$ изображения $\mathrm{AS}^{\prime}$ апертурной диафрагмы осветительной системы и фокусным расстоянием $f$ микрообъектива (рис. 3, $a$ ). При максимально раскрытой апертурной диафрагме угловая апертура $\theta$ освещающего объект поля совпадает с угловой апертурой входного зрачка микрообъектива $\theta_{m}$, $\theta=\theta_{m}$, и угловой спектр освещающего объект поля определяется полной числовой апертурой микрообъектива $N A=n_{i} \sin \left(\theta_{m}\right)$, где $n_{i}$ - показатель преломления иммерсии. Объем когерентности освещающего поля в этом случае в соответствии с (9) можно выразить через числовую апертуру микрообъектива:

$$
\begin{aligned}
V_{c} \approx & \frac{\pi}{2} \frac{\lambda_{0}^{2}}{\left(2+\frac{\Delta \lambda}{\lambda_{0}}\right)^{2}\left(\frac{N A}{n_{i}}\right)^{2}} \\
& \times \frac{\lambda_{0}}{\left[\left(2+\frac{\Delta \lambda}{\lambda_{0}}\right)-\left(2-\frac{\Delta \lambda}{\lambda_{0}}\right) \sqrt{1-\left(\frac{N A}{n_{i}}\right)^{2}}\right]} .
\end{aligned}
$$

Это выражение позволяет оценить объем когерентности освещающего объект поля в зависимости от $N A$ при разной ширине $\Delta \lambda$ частотного спектра поля в разных случаях, когда $l_{c} \ll \rho_{\|}$или $l_{c} \gg \rho_{\|}$, или $l_{c} \approx \rho_{\|}$.

В микроскопии прозрачных объектов на пропускание угловая апертура освещающего объект поля может быть как меньше $\theta<\theta_{m}$, так и больше $\theta>\theta_{m}$ угловой апертуры используемого микрообъектива (рис. $3, b$ ). При $\theta<\theta_{m}$ объем когерентности поля определяется угловой апертурой осветительной системы $\theta$ в соответствии с (9). При $\theta>\theta_{m}$ реальный объем когерентности освещающего поля также определяется угловой апертурой $\theta$, но поскольку апертура микрокобъектива $\theta_{m}$ меньше $\theta$, то оптическая система формирования изображения объекта воспринимает только часть освещающего объект поля, которая соответствует угловой апертуре $\theta \approx \theta_{m}$. Поэтому объем когерентности освещающего объект поля, который необходимо учитывать на практике, определяется полной числовой апертурой микрообъектива $N A$, и его следует рассчитывать с использованием выражения (11).

В изображающих оптических системах, в том числе и в микроскопии, формирование изображений может

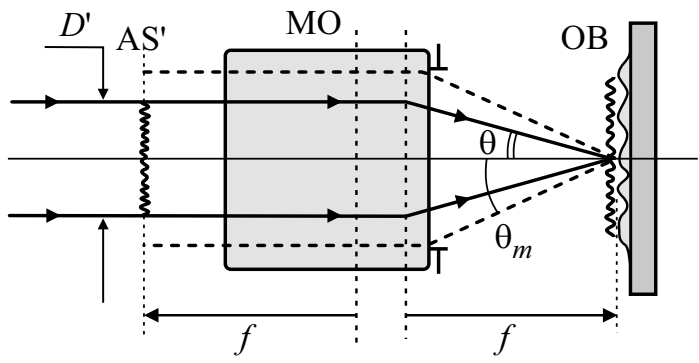

$a$

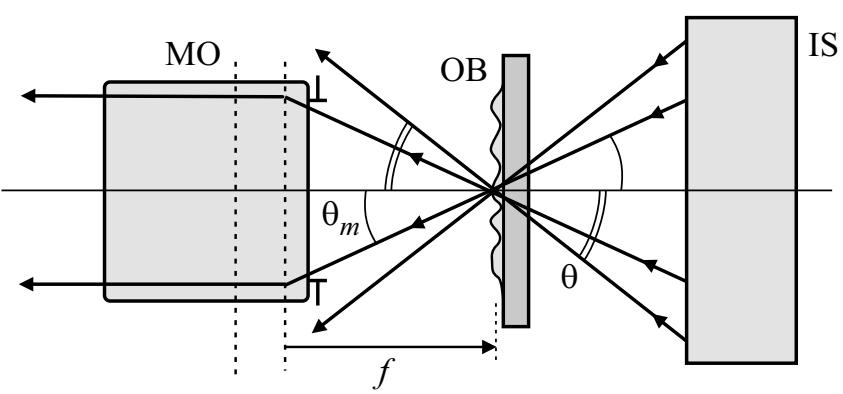

Рис. 3. Оптические системы освещения и формирования изображения в микроскопии отражающих $(a)$ и пропускающих $(b)$ объектов: $\mathrm{AS}^{\prime}$ - поле изображения апертурной диафрагмы осветительной системы микроскопа, $D^{\prime}$ - диаметр изображения апертурной диафрагмы, МО - микрообъектив микроскопа, OB - объект на предметном стекле, IS осветительная система микроскопа на пропускание.

носить когерентный, частично когерентный или почти некогерентный характер в зависимости оттого, разрешает, частично разрешает или почти совсем не разрешает оптическая система область пространственной когерентности поля в пространстве фокусировки этой системы. При этом пространственное разрешение микроскопа (поперечное и продольное при использовании дифракционно ограниченных микрообъективов) определяется числовой апертурой микрообъективов $[1,8]$. Из вышеприведенных суждений следует, что в значительной степени когерентное формирование изображения поверхности объекта и его внутренней структуры происходит при пространственном разрешении микроскопом области когерентности, когда угловая апертура освещающего объект поля существенно меньше угловой апертуры микрообъектива, $\theta \ll \theta_{m}$. При $\theta<\theta_{m}$ имеет место частичное разрешение области когерентности и соответственно частично когерентное формирование изображения. При $\theta \approx \theta_{m}$ и $\theta>\theta_{m}$ размеры области разрешения микроскопа примерно равны размерам области когерентности освещающего поля, воспринимаемого оптической системой микроскопа, и поэтому имеет место почти некогерентное формирование изображения. Когерентный и частично когерентный процессы формирования изображений выражаются в эффектах интерференции волн в изображении и соответственно искажении поля формируемого изображения по отношению к переотображаемому объектному полю, что 
необходимо учитывать при практическом использовании микроскопа.

\section{Условия доминирующего влияния углового или частотного спектров на продольную длину когерентности и влияния ширины частотного спектра на поперечную когерентность поля}

Рассмотренные выше соотношения между длиной временно́й когерентности $l_{c}$, зависящей от ширины частотного спектра волнового поля, и длиной продольной когерентности $\rho_{\|}$, зависящей от ширины углового спектра, $l_{c} \gg \rho_{\|}$и $l_{c} \ll \rho_{\|}$, определяют условия доминирующего влияния параметров частотного или углового спектров на результирующую продольную длину $L_{c}$ области когерентности поля. Однако для использования этих соотношений необходим их количественный характер, определяющий степень преобладания влияния на продольную когерентность одного спектра по сравнению с другим. Ниже рассмотрены такие количественные соотношения между этими длинами когерентности.

Выражения (4) и (5) определяют результирующую длину продольной когерентности $L_{c}$ волнового оптического поля в зависимости от ширины частотного $\Delta \lambda$ и углового $2 \theta$ спектров поля. Параметры частотного спектра задаются физической природой излучателей источника оптического поля и полагаются неизменными во всех точках пространства (дифракционный эффект изменения частотного спектра поля - эффект Вольфа $[55,56]$ - не учитывается), в то время как ширина углового спектра поля различна в различных точках пространства тех или иных оптических систем. Поэтому ниже рассматриваются условия ограничения значений угла $\theta$, определяющего ширину углового спектра, при заданных значениях $\lambda_{0}$ и $\Delta \lambda$ (или $l_{c}$ ). Продольная длина области когерентности волнового поля определяется только частотным спектром, когда выполняется условие $\rho_{\|} \gg l_{c}$. В этом случае можно записать

$$
L_{c} \geq \eta l_{c} \Leftrightarrow \frac{\rho_{\|} l_{c}}{\rho_{\|}+l_{c}} \geq \eta l_{c}
$$

где $\eta$ - положительное, близкое к единице, но не превосходящее ее число.

Выражение (12) преобразуется к следующему виду:

$$
l_{c} \leq \frac{1-\eta}{\eta} \rho_{\|}
$$

Из (13) и (3) можно получить выражение, ограничивающее сверху значения угла $\theta$, при котором влияние частотного спектра на продольную когерентность волнового оптического поля преобладает над влиянием углового спектра,

$$
\begin{aligned}
\theta & \leq 2 \arcsin \left(\sqrt{\frac{1-\eta}{2 \eta}} \sqrt{\frac{\lambda_{0}}{l_{c}}}\right) \\
& =2 \arcsin \left(\sqrt{\frac{1-\eta}{2 \eta}} \sqrt{\frac{\Delta \lambda}{\lambda_{0}}}\right) \approx \sqrt{2 \frac{1-\eta}{\eta}} \sqrt{\frac{\Delta \lambda}{\lambda_{0}}} .
\end{aligned}
$$

Поскольку в рассматриваемом случае углы $\theta$ достаточно малы и ограничены по величине снизу нулем, то в (14) использовано параксиальное приближение.

Аналогичным образом можно получить соотношение между параметрами углового и частотного спектров, при котором продольная длина области когерентности волнового поля в области наблюдения определяется преимущественно угловым спектром:

$$
L_{c} \geq \mu \rho_{\|} \Leftrightarrow \frac{\rho_{\|} l_{c}}{\rho_{\|}+l_{c}} \geq \mu \rho_{\|},
$$

где $\mu$ - также положительное, близкое к единице, но не превосходящее ее число.

Выражение (15) преобразуется к следующему виду:

$$
l_{c} \geq \frac{\mu}{1-\mu} \rho_{\|}
$$

Из (16) можно получить выражение, ограничивающее снизу значения угла $\theta$, при котором влияние углового спектра на продольную когерентность волнового оптического поля преобладает над влиянием частотного спектра,

$$
\begin{aligned}
\theta & \geq \arcsin \left(\sqrt{\frac{\mu}{2(1-\mu)}} \sqrt{\frac{\lambda_{0}}{l_{c}}}\right) \\
& =2 \arcsin \left(\sqrt{\frac{\mu}{2(1-\mu)}} \sqrt{\frac{\Delta \lambda}{\lambda_{0}}}\right) .
\end{aligned}
$$

Сверху значения угла $\theta$ ограничиваются величиной $\pi / 2$.

Влияние обоих спектров, частотного и углового, на продольные когерентные свойства волнового оптического поля будет совместным для точек пространства, в которых ширина углового спектра определяется значениями углов $\theta$ из интервала

$$
\sqrt{2 \frac{1-\eta}{\eta}} \sqrt{\frac{\Delta \lambda}{\lambda_{0}}}<\theta<2 \arcsin \left(\sqrt{\frac{\mu}{2(1-\mu)}} \sqrt{\frac{\Delta \lambda}{\lambda_{0}}}\right) .
$$

Если для коэффициентов $\eta$ и $\mu$ ограничиться значениями $\eta=\mu=0.9$, что означает, что величины $L_{c}$ в рассмотренных крайних случаях отличаются от значений $l_{c}$ и $\rho_{\|}$не более чем на $10 \%$, то условия $(13)$ и $(16)$ принимают соответственно вид

$$
\begin{gathered}
l_{c} \leq \rho_{\|} / 9, \\
l_{c} \geq 9 \rho_{\|} .
\end{gathered}
$$



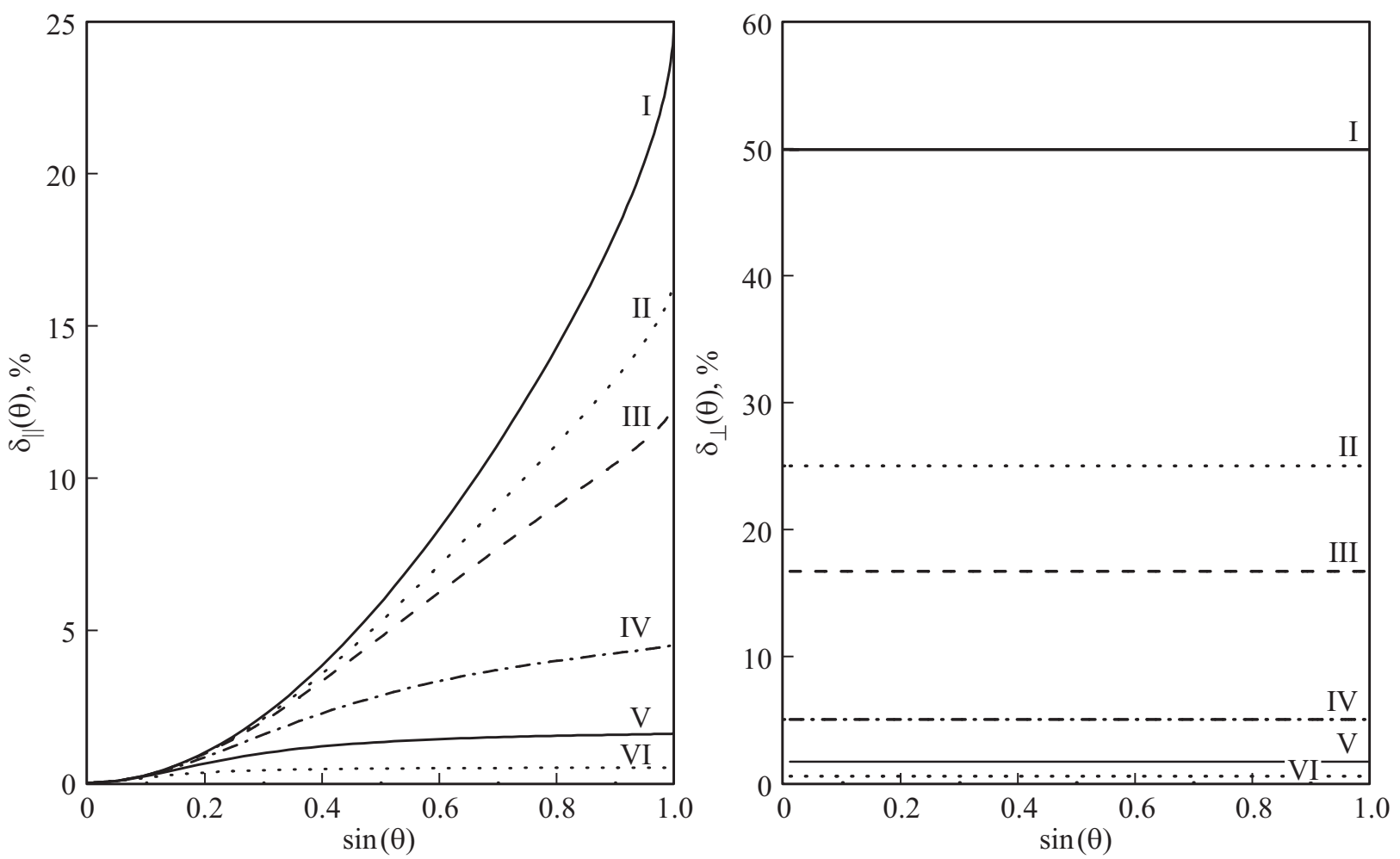

Рис. 4. Зависимость относительных погрешностей $\delta_{\|}(a)$ и $\delta_{\perp}(b)$ от $\sin (\theta)$ при определении длины продольной когерентности $L_{c}$ и длины поперечной когерентности $R_{\perp}$ по приближенным формулам при различных значениях отношения $l_{c} / \lambda_{0} \approx \lambda_{0} / \Delta \lambda: 1(\mathrm{I})$, 2 (II), 3 (III), 10 (IV), 30 (V), 100 (VI).

Таким образом, влияние углового или частотного спектров волнового оптического поля на длину продольной когерентности в рассматриваемой области пространства становится преобладающим, если одна из величин, $l_{c}$ или $\rho_{\|}$, превышает другую примерно в 10 раз (на порядок).

Рассмотренный выше анализ проводился без учета в (4) и (5) сомножителя $\cos ^{2}(\theta / 2)$, т.е. при условии $\Delta \lambda \ll \lambda_{0}$. Для оценки влияния этого сомножителя и уточнения данного условия необходимо учесть относительную погрешность, возникающую при использовании приближенной формулы

$$
\delta_{\|}\left(\theta, l_{c}\right)=\left(1-\frac{\rho_{\|} \cos ^{2}(\theta / 2)+l_{c}}{\rho_{\|}+l_{c}}\right) 100 \%
$$

На рис. 4, $a$ приведены графики зависимости величины $\delta_{\|}$от $\sin (\theta)$ при различных значениях отношения $l_{c} / \lambda_{0} \approx \lambda_{0} / \Delta \lambda$. Графики на рис. 4, $a$ показывают, что значение величины $\delta_{\|}$растет при увеличении ширины углового спектра $2 \theta$ (и соответственно увеличении величины $\sin (\theta))$, которая, например, в высокоразрешающей микроскопии определяет числовую апертуру $N A$ освещающего объект поля), однако для источников $\mathrm{c} l_{c} / \lambda_{0}>10$ $\left(\Delta \lambda<0.1 \lambda_{0}\right)$ не превышает $5 \%$. В эту категорию оптических источников попадают светодиоды окрашенного излучения, суперлюминесцентные диоды и лазеры различных типов, в частности полупроводниковые лазеры.
Для подобных источников вполне оправдано применение упрощенного вида формул (4) и (5) для определения длины $L_{c}$ когерентности излучения в продольном направлении.

Однако для широкополосных источников, например источников белого света с $l_{c} / \lambda_{0} \approx 3\left(\Delta \lambda \approx 0.33 \lambda_{0}\right)$, таких как лампы накаливания, белые светодиоды, а также для естественного (солнечного) света видимого диапазона, при формировании поля с большой шириной углового спектра (при $\theta \rightarrow \pi / 2$ ), например в микроскопии при использовании объективов с большой числовой апертурой $(N A \rightarrow 1)$, значение $\delta_{\|}$может существенно превышать 5\%. Так, например, для указанного отношения длины временной когерентности $l_{c}$ и средней длины волны $\lambda_{0}$ относительная погрешность $\delta_{\|} \approx 9 \%$ возникает при $N A=0.8$, а при $N A=1$ величина $\delta_{\|}$достигает значения $12.5 \%$.

Еще бо́льшие погрешности могут возникать при оценке продольной длины когерентности $L_{c}$ волнового поля фемтосекундного спектрального суперконтинуума [57-60], для которого отношение средней длины волны к ширине спектра оказывается равным или даже меньше единицы, $l_{c} / \lambda_{0} \approx \lambda_{0} / \Delta \lambda \leq 1$. Такие количественные оценки важны, в частности, при использовании такого излучения в полнопольной оптической когерентной томографии [15,61-63]. Так, например, при $l_{c} / \lambda_{0} \approx 1$ относительная погрешность $\delta_{\|}$при $N A=0.8$ состав- 
ляет $13 \%$, а при $N A=1$ величина $\delta_{\|}$уже достигает значения $24 \%$ (рис. $4, a$ ). Таким образом, в ряде случаев для более точного определения значения продольной длины когерентности $L_{c}$ поля частотно широкополосного источника с широким угловым спектром следует учитывать в формулах (4) и (5) сомножитель $\cos ^{2}(\theta / 2)$, а для узкополосных полей с $\Delta \lambda<0.1 \lambda_{0}$ этот сомножитель можно фактически не учитывать при любых значениях угла $\theta$.

Аналогичным образом можно сделать оценку приближения для длины поперечной когерентности $R_{\perp} \approx \rho \perp$ при $\Delta \lambda \ll \lambda_{0}$. Для относительной погрешности в этом случае можно получить выражение

$$
\begin{aligned}
\delta_{\perp}\left(\theta, l_{c}\right) & =\left|\frac{R_{\perp}-\rho_{\perp}}{R_{\perp}}\right| 100 \% \\
& =\frac{\rho_{\perp} \sin (\theta)}{l_{c}} 100 \%=\frac{\lambda_{0}}{2 l_{c}} 100 \% .
\end{aligned}
$$

Выражение (22) показывает, что относительная погрешность $\delta_{\perp}$ не зависит от ширины углового спектра поля и целиком определяется отношением $\lambda_{0} / l_{c} \approx \Delta \lambda / \lambda_{0}$, при этом $\delta_{\perp}$ увеличивается с увеличением $\Delta \lambda$ (рис. $4, b)$. Как и в случае с $\delta_{\|}$, относительная погрешность $\delta_{\perp}$ не превышает $5 \%$ для источников с $l_{c} / \lambda_{0}>10\left(\Delta \lambda<0.1 \lambda_{0}\right)$, а для источников белого света c $l_{c} / \lambda_{0} \approx 3\left(\Delta \lambda \sim 0.33 \lambda_{0}\right)$ достигает $17 \%$. При этом для фемтосекундного спектрального суперконтинуума уже при $l_{c} / \lambda_{0} \approx \lambda_{0} / \Delta \lambda \approx 1$ эта погрешность достигает 50\%. Поэтому для полей таких источников длину поперечной когерентности $R_{\perp}$ следует определять с помощью выражения (7), учитывающего влияние ширины частотного спектра.

\section{Заключение}

Объем когерентности частично когерентного волнового поля в общем случае определяется параметрами и углового, и частотного спектров волнового поля в той области пространства, где рассматривается объем когерентности поля. В разных областях пространства в силу закономерностей дифракционного процесса распространения волнового поля и преобразования его оптическими системами угловые и частотные параметры поля могут быть разными и, следовательно, разным объем когерентности поля. Поперечные размеры области когерентности зависят в основном от ширины углового спектра поля. Однако для частотно широкополосного поля, когда ширина спектра сравнима с его средней частотой или превышает ее, как в поле фемтосекундного спектрального суперконтинуума, поперечная длина когерентности оказывается зависимой еще и от ширины частотного спектра. Продольные размеры области когерентности могут зависеть как от ширины частотного спектра, так и от ширины углового спектра поля или совместно и от ширины частотного, и от ширины углового спектров поля.
Такие представления о зависимости объема когерентности от ширины частотного и ширины углового спектров поля эффективны для практического анализа поля в оптических системах, поскольку и частотный, и угловой спектры поля и их параметры являются измеряемыми характеристиками поля. Однако с обобщенной теоретической точки зрения объем когерентности определяется пространственной (поперечной и продольной) когерентностью поля, которая, как показано в [33,34], в соответствии с теоремой Винера-Хинчина для пространственной когерентности зависит от параметров спектров поперечных и продольных пространственных частот волнового поля. Эти пространственные спектры поля, в свою очередь, определяются совместно и угловым спектром поля, и его частотным спектром. Поэтому в общем случае можно утверждать, что объем когерентности волнового поля полностью определяется его пространственным спектром, зависящим и от частотного, и от углового спектров поля.

Оценка и учет объема когерентности волнового поля необходимы при практическом использовании оптических методов формирования и визуализации изображений, интерференционных измерений поверхностной и объемной структуры микрообъектов, в высокоразрешающей оптической микроскопии, включая интерференционную микроскопию и полнопольную когерентную томографию, в оптике рассеивающих сред, включая оптические исследования биологических тканей и материаловедение, в том числе при использовании терагерцового излучения [64].

Авторы выражают благодарность Л.А. Максимовой за помощь в численном моделировании спекл-картин. Работа выполнена при поддержке Российского научного фонда (проект № 16-19-10528).

\section{Список литературы}

[1] Born M., Wolf E. Principles of Optics. Cambridge University Press, 2002. 994 р.; Борн М., Воль $\oint$ Э. Основы оптики. М.: Наука, 1973. 720 с. doi: 10.1017/CВO9781139644181

[2] Mandel L., Wolf E. Optical Coherence and Quantum Optics. NY: Cambridge University Press, 1995. 1166 p.; Мандель Л., Вольф Э. Оптическая когерентность и квантовая оптика. М.: Физматлит, 2000. 896 с. doi: 10.1017/CBO9781139644105

[3] Wolf E. Introduction to the Theory of Coherence and Polarization of Light. Cambridge University Press, 2007. $236 \mathrm{p}$.

[4] Goodman J.W. Statistical Optics. Wiley, 2000. 567 p.; Гудмен Джс. Статистическая оптика. М.: Мир, 1988. 528 с. doi: $10.1063 / 1.2815179$

[5] Ахманов С.А., Дьяков Ю.Е., Чиркин А.С. Статистическая радиофизика и оптика. Случайные колебания и волны в линейных системах. М.: Физматлит, 2010. 428 с.

[6] Hariharan P. Basics of Interferometry. Academic Press, 2010. 248 p. doi: $10.1063 / 1.2808787$ 
[7] Hitzenberger C.K. Low-coherence Interferometry. Handbook of Visual Optics, Volume Two: Instrumentation and Vision Correction. CRC Press, 2017. P. 37. doi: 10.1201/9781315373027-4

[8] Marechal A., Françon M. Diffraction, Structure des Images; Influence de la Coherence de la Lumiere. Paris: Masson, 1960; Марешаль А., Франсон М. Структура оптического изображения. Дифракционная теория и влияние когерентности света. М.: Мир, 1964. 295 с.

[9] Thompson B.J. // Progress in Optics. 1969. V. 7. P. 169. doi: 10.1016/S0079- 6638(08)70595-X

[10] De Groot P. // Advanced in Optics and Photonics. 2015. V. 7. N 1. P. 1. doi: 10.1364/AOP.7.000001

[11] Abdulhalim I. // Ann. Phys. 2012. V. 524. N 12. P. 787. doi: 10.1002/andp.201200106

[12] Dubois F., Callens N., Yourassowsky C., Hoyos M., Kurowski P., Monnom O. // Appl. Opt. 2006. V. 45. N 5. P. 864. doi 10.1364/AO.45.000864

[13] Каленков Г.С., Каленков С.Г., Штанько А.Е. // Квант. электрон. 2015. Т. 45. № 4. С. 333; Kalenkov G.S., Kalenkov S.G., Shtanko A.E. // Quant. Electron. 2015. V. 45. N 4. P. 333. doi 10.1070/QE2015v045n04ABEH015584

[14] Drexler W., Fujimoto J.G. Optical Coherence Tomography: Technology and Applications. Springer, 2015. 2571 p. doi: 10.1007/978-3-319-06419-2

[15] Dubois A. (ed.) Handbook of Full-Field Optical Coherence Microscopy: Technology and Applications. Singapore: Pan Stanford Publishing, 2016. 800 p. doi: $10.4032 / 9789814669177$

[16] Dainty J.C. (ed.) Laser Speckle and Related Phenomena. Springer Science \& Business Media, 2013. V. 9. 286 p. doi: $10.1007 \mid 978-3-662-43205-1$

[17] Goodman J.W. Speckle Phenomena in Optics: Theory and Applications. Englewood: Roberts \& Company, Publishers, 2007. $387 \mathrm{p}$.

[18] Будаговский, А.В. // Квант. электрон. 2005. Т. 35. № 4. C. 369; Budagovsky A.V. // Quant. Electron. 2005. V. 35. N 4. P. 369. doi: 10.1070/QE2005v035n04ABEH002837

[19] Будаговский А.В., Соловых Н.В., Будаговская О.Н., Будаговский И.А. // Квант. электрон. 2015. Т. 45. № 4. C. 351; Budagovsky A.V., Solovykh N.V., Budagovskaya O.N., Budagovsky I.A. // Quant. Electron. 2015. V. 45. N 4. P. 351. doi: 10.1070/QE2015v045n04ABEH015594

[20] Françon M., Mallick S. // Appl. Opt. 1967. V. 6. N 5. P. 873. doi: 10.1364/AO.6.000873

[21] Сороко Л.М. Основы голографии и когерентной оптики. М.: Наука, 1971. 686 с.; Soroko L.M. Holography and Coherent Optics. NY.: Plenum Press, 1980. 818 c. doi: 10.1007/978-1-4684-3420-0

[22] Zarubin A.M. // Opt. Commun. 1993. V. 100. N 5. P. 491.

[23] Wolf E. // Opt. Lett. 1994. V. 19. N 23. P. 2024. doi: 10.1364/OL.19.002024

[24] Ryabukho V.P., Klimenko I.S., Golubentseva L.I. // Proc. SPIE. 1994. V. 2340. P. 513.

[25] Rosen J., Yariv A. // Opt. Commun. 1995. V. 117. N 1-2. P. 8. doi: 10.1016/0030- 4018(95)00086-N

[26] Rosen J., Yariv A. // J. Opt. Soc. Am. A. 1996. V. 13. № 10. P. 2091. doi: 10.1364/JOSAA.13.002091

[27] Рябухо В.П., Лякин Д.В., Лобачев М.И. // Опт. и спектр. 2004. T. 97. № 2. C. 319; Ryabukho V.P., Lyakin D.V., Lobachev M.I. // Opt. Spectrosc. 2004. V. 97. N 2. P. 299. doi: $10.1134 / 1.1790649$
[28] Ryabukho V.P., Lyakin D.V., Lobachev M.I. // Opt. Lett. 2004. V. 29. N 7. P. 667. doi: $10.1364 /$ OL.29.000667

[29] Рябухо В.П., Лякин Д.В. // Опт. и спектр. 2005. Т. 98. В. 2. C. 309; Ryabukho V.P., Lyakin D.V. // Opt. Spectrosc. 2005. V. 98. N 2. P. 273. doi: $10.1134 / 1.1870071$

[30] Abdulhalim I. // J. Opt. A: Pure Appl. Opt. 2006. V. 8. N 11. P. 952. doi: 10.1088/1464-4258/8/11/004.

[31] Liu H., Han S. // Opt. Lett. 2008. V. 33. N 8. P. 824. doi: 10.1364/OL.33.000824

[32] Cai Y., Dong Y., Hoenders B.J. // JOSA A. 2012. V. 29. N 12. P. 2542. doi: 10.1364/JOSAA.29.002542

[33] Рябухо В.П., Лякин Д.В., Лычагов В.В. // Опт. и спектр. 2009. T. 107. B. 2. C. 300; Ryabukho V.P., Lyakin D.V., Lychagov V.V. // Opt. Spectrosc. 2009. V. 107. N 2. P. 282. doi: 10.1134/S0030400X09080190

[34] Ryabukho V.P., Lyakin D.V., Grebenyuk A.A., Klykov S.S. // J. Optics. 2013. V. 15. N 2. P. 025405. doi: 10.1088/20408978/15/2/025405

[35] Рябухо В.П., Кальянов А.Л., Лычагов В.В., Лякин Д.В. // Опт. и спектр. 2010. Т. 108. В. 6. С. 1032; Ryabukho V.P., Kal'yanov A.L., Lyakin D.V., Lychagov V.V. // Opt. Spectrosc. 2010. V. 108. N 6. P. 979. doi: 10.1134/S0030400X1006024X

[36] Martienssen W., Spiller E. //American J. Physics. 1964. V. 32. N 12. P. 919. doi: $10.1119 / 1.1970023$

[37] Okamoto T., Asakura T. // Progress in Optics. 1995. V. 34. P. 183. doi: 10.1016/S0079-6638(08)70326-3

[38] Rabal H.J., Braga R.A., jr. Dynamic Laser Speckle and Applications. CRC Press, 2008. 282 p. doi: 10.1201/9781420060164

[39] Козлов С.A. // Соросовский образовательный журнал. 2001. T. 7. № 2. C. 76.

[40] Рябухо В.П. // Физическое образование в вузах. 2008. Т. 14. № 3. C. 88.

[41] Рытов С.М., Кравцов Ю.А., Татарский В.И. Введение в статистическую радиофизику. Ч. 2. Случайные поля. М.: Наука, 1978. 464 c.; Rytov S.M., Kravtsov Y.A., Tatarskii V.I. Principles of Statistical Radiophysics 3. Elements of Random Fields. Berlin, Heidelberg: Springer-Verlag, 1989. 239 p. doi: 10.1007/978-3-642-72685-9

[42] Ryabukho V.P., Lyakin D.V., Lobachev M.I. // Opt. Lett. 2005. V. 30. N 3. P. 224. doi: $10.1364 /$ OL.30.000224

[43] Рябухо В.П., Лякин Д.В., Лычагов В.В. // Опт. и спектр. 2006. T. 100. B. 5. C. 788; Ryabukho V.P., Lyakin D.V., Lychagov V.V. // Opt. Spectrosc. 2006. V. 100. N 5. P. 724. doi: 10.1134/S0030400X06050146

[44] Dubois A., Vabre L., Boccara A.C., Beaurepaire E. // Appl. Opt. 2002. V. 41. N 4. P. 805. doi: 10.1364/AO.41.000805

[45] Zeylikovich I. // Appl. Opt. 2008. V. 47. N 12. P. 2171. doi: 10.1364/AO.47.002171

[46] Srivastava V., Nandy S., Singh Mehta D. // Appl. Phys. Lett. 2013. V. 103. N 10. P. 103702. doi: $10.1063 / 1.4820350$

[47] Ahmad A., Srivastava V., Dubey V., Mehta D.S. // Appl. Phys. Lett. 2015. V. 106. N 9. P. 093701. doi: 10.1063/1.4913870

[48] Safrani A., Abdulhalim I. // Appl. Opt. 2011. V. 50. N 18. P. 3021. doi: 10.1364/AO.50.003021

[49] Safrani A., Abdulhalim I. // Opt. Lett. 2012. V. 37. N 4. P. 458. doi: 10.1364/OL.37.000458

[50] Rosen J., Takeda M. // Appl. Opt. 2000. V. 39. N 23. P. 4107. doi: 10.1364/AO.39.004107

[51] Pavliček P., Halouzka M., Duan Z., Takeda M. // Appl. Opt. 2009. V. 48. N 34. P. H40. doi: 10.1364/AO.48.000H40 
[52] Gao W. // J. Modern Optics. 2015. V. 62. N 21. P. 1764. doi: 10.1080/09500340.2014.952689

[53] Gao W. // J. Microscopy. 2016. V. 261. N 3. P. 199. doi 10.1111/jmi.12333

[54] Лякин Д.В., Рябухо П.В., Рябухо В.П. // Опт. и спектр. 2017. T. 122. B. 2. C. 336; Lyakin D.V., Ryabukho P.V., Ryabukho V.P. // Opt. Spectrosc. 2017. V. 122. N 2. P. 329. doi 10.1134/S0030400X17020175

[55] Wolf E. // Phys. Rev. Lett. 1986. V. 56. N 13. P. 1370. doi: 10.1103/PhysRevLett.56.1370

[56] Дьяков Ю.Е. // Квант. электрон. 1993. Т. 20. № 11. С. 1068; D'yakov Yu. E. // Quant. Electron. 1993. V. 23. N 11. P. 931. doi: 10.1070/QE1993v023n11ABEH003225

[57] Желтиков А.М. // УФН. 2006. Т. 176. № 6. С. 623; Zheltikov A.M. // Phys. Usp. 2006. V. 49. P. 605. doi: 10.1070/PU2006v049n06ABEH005975

[58] Минкович В.П., Сотский А.Б., М. Vаса P.G., Дзен И.С., Сотская Л.И. // Журн. прикл. спектр. 2016. Т. 83. № 2. C. 216; Minkovich V.P., Sotsky A.B., Vaca Pereira M.G., Dzen I.S., Sotskaya L.I. // J. Appl. Spectroscopy. 2016. V. 83. I. 2. P. 198. doi: 10.1007/s10812-016-0269-2

[59] Чекалин С.В., Компанеи, В.О., Дормидонов А.Е., Залозная Е.Д., Кандидов В.П. // Квант. электрон. 2017. T. 47. № 3. C. 252; Chekalin S.V., Kompanets V.O., Dormidonov A.E., Zaloznaya E.D., Kandidov V.P. // Quant. Electron. 2017. V. 47. I. 3. P. 252. doi: 10.1070/QEL16289

[60] Боримова А.А., Цыпкин А.Н., Путилин С.Э., Беспалов В.Г., Козлов С.А. // Оптический журнал. 2017. Т. 84. № 6. C. 10; Borimova A.A., Tsypkin A.N., Putilin S.E., Bespalov V.G., Kozlov S.A. // J. Optical Technology. 2017. V. 84. N 6. P. 368 . doi: $10.1364 /$ JOT. 84.000368

[61] Hartl I., Li X.D., Chudoba C., Ghanta R.K., Ko T.H., Fujimoto J.G., Ranka J.K., Windeler R.S. // Opt. Lett. 2001. V. 26. N 9. P. 608. doi: 10.1364/OL.26.000608

[62] Povazay B., Bizheva K., Unterhuber A., Hermann B., Sattmann H., Fercher A.F., Drexler W., Apolonski A., Wadsworth W.J., Knight J.C., Russell P.S., Vetterlein M., Scherzer E. // Opt. Lett. 2002. V. 27. N 20. P. 1800. doi: 10.1364/OL.27.001800

[63] Humbert G., Wadsworth W., Leon-Saval S., Knight J., Birks T., Russell P.St.J., Lederer M., Kopf D., Wiesauer K., Breuer E., Stifter D. // Optics Express. 2006. V. 14. N 4. P. 1596. doi: 10.1364/OE.14.001596

[64] Розанов Н.Н., Сочилин Г.Б., Федоров С.В., Шацев А.Н., Малевич В.Л., Синицын Г.В. // Опт. и спектр. 2017. Т. 123. B. 1. C. 132; Rosanov N.N., Sochilin G.B., Fedorov S.V., Shatsev A.N., Malevich V.L., Sinitsyn G.V. // Opt. Spectrosc. 2017. V. 123. N 1. P. 146. doi: $10.7868 /$ S0030403417070212 